\title{
Comparison between the right and left radial approach in coronariography
}

\begin{abstract}
In the 1980s, Campeu and Kiemeneij introduced the radical approach for angiography and angioplasty, respectively, in 2017 Kiemeneij described the site of radial distal access to the snuffbox, as the viability and safety of the left approach, either conventional or distal, remains a concern and there are no studies evaluating comfort yet. We randomly assigned 55 patients ( 9 were excluded), to either left radial access or right radial access ( 27 pts vs $28 \mathrm{pts}$ ). The primary end point was patient's comfort, contrast volume, distance between first operator and patient, number of angiography catheters, fluoroscopy time. As secondary safety endpoints we include radial spasm and procedure related bleeding. Statistical analysis was done with descriptive statistics, $\mathrm{T}$ student for quantitative variables and square chi for qualitative variables. From January 2019 to September 2019 we enrolled 64 patients, (9 were excluded) Stable coronary artery disease was the most common indication for angiography ( $60 \%$ left vs $43 \%$ right). Both access were perceived as comfortable ( 4.29 left vs 4.18 right $\mathrm{P}=0.549)$, the amount of contrast volume used was $(103 \pm 85 \mathrm{ml}$ vs $88 \pm 55 \mathrm{ml}$, $\mathrm{P}=0.436$, distance between intensifier-researcher $(47.6 \mathrm{~cm} \pm 4.6$ right vs $47.7 \mathrm{cms} \pm 4.8$ left, $\mathrm{p}=0.941)$. In safety endpoints the presence of bleeding was $7 \%$ vs $7 \%(\mathrm{P}=0.99)$ and radial artery spasm $26 \%$ and $11 \%$ respectively $(\mathrm{P}=0.177)$. In terms of comfort and safety there is no difference between left and right radial access, both access sites can be done with femoral and radial dedicated catheters in stable coronary disease and acute coronary syndrome coronary.
\end{abstract}

Keywords: coronary angiography, hemostasis, arteriovenous fistulas, fluoroscopy, coronary artery, revascularization, ischemic heart disease
Volume 13 Issue 4 - 2020

\author{
Cázares-Díazleal AC,' Dorantes-García \\ J, ${ }^{2}$ Kiamco-Castillo R, ${ }^{3}$ Payro-Ramirez G, ${ }^{2}$ \\ Betuel-Ivey J, ${ }^{4}$ Lozano-Sabido $E,{ }^{3}$ Arce- \\ González J' \\ 'Department of Cardiology and Interventional Cardiology, \\ Hospital Español de Mexico, Mexico \\ ${ }^{2}$ Department of Cardiology and Interventional Cardiology, \\ Instituto Nacional de Ciencias Medicas y Nutrición Salvador \\ Zubiran, Mexico \\ ${ }^{3}$ Department of Cardiology, Hospital Español de Mexico, Mexico \\ ${ }^{4}$ Department of Cardiology, Centro Medico Nacional S XXI \\ Hospital Cardiologia, Mexico
}

\section{Correspondence: Cázares-Díazleal AC, Department of Cardiology and Interventional Cardiology Hospital Español de Mexico, Email a.czdiazleal@gmail.com}

Received: July 02, 2020 | Published: August 3I, 2020

\section{Introduction}

Since the 1940 s, various methods for creating vascular access have been described. In 1929 Werner Forssman who at the time was a surgical resident performs the first catheterization using a urinary catheter. ${ }^{1}$ In 1941 Fariñas described the passage of a urinary catheter through a trocar placed in the femoral artery to perform an aortography. ${ }^{2}$ In1947 Radner through a dissecting artery exposed the radial artery and performed a vertebral angiography. In 1949 Jönsson performed an aortography performing a puncture at the common carotid artery using a blunt cannula with a needle the cannula was inserted through a silver wire. In 1953 Dr. Sven Ivar Seldinger described a technique which consisted of performing a puncture using a stylet, subsequently a $3 \mathrm{~cm}$ flexible guidewire was inserted, the needle was withdrawn and the vessel was compressed, at the meantime a catheter was passed through the flexible guide wire through the puncture site and finally the guidewire was withdrawn guide. ${ }^{3}$ Dotter and Judkins (who described femoral access for coronary angiography) in 1964 performed the first transluminal angioplasty in an 83-year-old patient, showing a decrease in temperature and pain of a pelvic limb and who refused to be amputated, they used a teflon-coated catheter to dilate the stenotic area. ${ }^{4,5}$ In 1977 Dr. Andreas Gruetzing performed the first successful coronary angioplasty in a human being, a ballon was mounted at his catheter.

Since Judkins' description of femoral access, this has become the standard for performing coronary angiography and angioplasty. The common femoral artery is considered the safest arterial puncture site since the path of this vessel passes over a bone structure that facilitates compression for hemostasis. However, this type of access is related to complications such as the formation of pseudoaneurysms, arteriovenous fistulas or distal thrombosis. ${ }^{6,7}$ These complications have been shown to have a prognostic impact on the patient, however, in various studies it has a low incidence of $2.4 \%$, in the setting of primary coronary angioplasty. ${ }^{8}$

In 1989 Campeau at the Montreal Heart Institute described radial access in 100 patients programmed for elective coronarography using a $5 \mathrm{Fr}$ sheath, in 10 patients radial artery puncture could not be performed and in 2 patients coronary artery cannulation was not possible, in the original technique description the left arm was abducted at a $70^{\circ}$ angle with the wrist in hyperextension For left radial access, an $18 \mathrm{G}$ needle was used, using a posterior puncture technique for large arteries and anterior wall for small arteries, a $23 \mathrm{cms} 5 \mathrm{Fr}$ sheath was used of $23 \mathrm{cms} .^{9}$

In 1995 Ferdinand Kiemeneij described the technique for radial coronary angioplasty placing the thoracic limb in $70^{\circ}$ of abduction with the wrist in hyperextension using a $22 \mathrm{G}$ needle and puncturing the anterior arterial wall, thereafter the application of this technique has gone from being one more option to the first access route used in primary angioplasty. ${ }^{10}$ Recently in 2017 Kiemeneij described the left radial access technique through the anatomic snuff box in 70 patients as a possible and safe technique with the advantage of using femoral angiographic catheters that follow the "natural" route through the left aortic arch, In addition, puncturing at the distal level reduces the risk of occlusion of the palmar arch. ${ }^{11}$ There have been recent publications of single center's experience that describes the use of left distal radial artery with good results with no equipment or investment necessary to support left arm, Nairoukh reported that distal radial diameter is $80 \%$ 
smaller, he described variations among different countries, the largest diameter was found in Korean patients and the smaller in patients from Singapore. ${ }^{12} \mathrm{Kim}$ et al found that left distal radial access was feasible and the success rate of radial artery cannulation was $88 \%$ with minor vascular complications such as forearm swelling and bruises. ${ }^{13}$ Mizuguchi et al in Japan made a multicentric study aimed to investigate radial patency and hemorrhagic events by performing vascular ultrasound before and after interventional procedure, they found no bleeding complications and a low incidence of radial artery occlusion. ${ }^{14}$ Despite being a safe procedure there are reports of serious complications such as Koutouzis reported the first case of hand hematoma which extended distal to the sheath insertion site, showing a rare but dangerous complication, highlighting the importance of performing a good and careful technique. ${ }^{15}$

The use of radial access in Mexico is the method of choice in angioplasty according to the revascularization guidelines published this year. Applied in the right patient means fewer complications. However, only a few radial catheter options are available in our country, in addition to the fact that the conventional procedure requires placing the patient in a position that is often referred to as uncomfortable. On the other hand, the left radial access supposes less time of radiation exposure, greater patient comfort and fewer embolic complications and vascular with the use of conventional catheters. Also, this technique should be more comfortable since the patient's left upper limb may be flexed towards the line medium and not fixed in supination and hyperextension.

\section{Methods}

\section{Primary outcomes}

a. Compare left and right radial approach based on

b. Patient comfort

c. Amount of contrast used between left and right radial access

d. Distance between the fluoroscope and the operator.

e. Number and type of catheters used for left and right radial access.

f. Time for coronary angiography.

\section{Secondary outcomes}

a. Assess presence of major bleeding defined by BARC scale comparing radial access right and left.

b. Presence of spasm

\section{Methodology}

A. Design

a. Analytical

b. Comparative

c. Cross sectional

d. Prospective

e. Multicenter

f. Controlled clinical trial

B. Population universe
Patients undergoing elective coronary angioplasty in the Hospital Español of Mexico and the "Instituto Nacional de Ciencias Medicas y Nutrición Salvador Zubirán" in the period of time between January 2019-September 2019.

\section{Study population}

Patients requiring coronary angiography, which are hemodynamically stable and having favorable characteristics for radial access. Stratified randomization by center in blocks of 10 IV.B1 I.

\section{Inclusion criteria}

a. Patients over 18years

b. Suspected ischemic heart disease.

c. Indication of preoperative coronary angiography.

d. Coronary angiography with probable percutaneous transluminal coronary angioplasty in Acute Coronary syndrome

\section{E. Exclusion criteria}

a. Patients with Renal Insufficiency

b. Barbeu C or D patients

\section{F. Elimination criteria}

a. Patients who refuse to participate in the protocol.

b. Patients in whom it is decided to use the femoral approach from the beginning.

c. Patients suffering from Raynaud's Syndrome.

Before each procedure an envelope was opened this contained the assigned access, an informed consent and data collection sheet. Each patient received information about data collection, technical aspects, risks, and the advantages of participating in the study, and the informed consent was signed. Data collection started with the patient's perception of comfort in relation to the position of the access site using a Likert scale (5 totally agree, 4 agree, 3 indifferent, 2 disagree, 1 totally disagree). Subsequently, distance between the fluoroscope and the operator's left shoulder was measured in centimeters using a tape. Once having knowledge of the assigned approach to carry out, the first operator freely chose the puncture site either at the wrist level or in the anatomic snuff box. Once the access site was obtained, the fluoroscopy time was recorded by the cath lab technician, data related to coronary angiography was collected such as type of angiographic catheter, if the patient had some anatomical variation such as loops, variations in the morphology of the aortic arch, variations in the position of the coronary ostiums or abnormal origins this was reported on the collection sheet as abnormal anatomy, otherwise as normal anatomy; if the patient presented radial artery spasm, it was recorded on the data collection sheet as well. At the end of the procedure, the participant was asked on comfort perception again.

Presence of bleeding after the procedure or until hospital discharge, was classified according to the Bleeding scale Academic Research Consortium from 0 to $5(0=$ No bleeding, $1=$ Bleeding that does not merit medical care, $2=$ Bleeding that merits studies and hospitalization, $3=$ requires surgical intervention to be controlled, $4=$ Surgery related bleeding of bridges, $5=$ Fatal leading to death). 
For the purpose of comparing comfort in patients with a right vs. left radial approach, a comparison of means (the group "Radial right" vs. "Radial left" was compared), the dependent variable was the degree of comfort according to the Likert scale, where the highest convenience or comfort was coded with a higher numerical value; (see annexes for more details).

\section{Statistic analysis}

a. Firstly, an exploratory analysis of the database was done to determine whether missing values existed.

b. Subsequently descriptive statistics was performed: the information was summarized with mean and standard deviation and interquartile range for the quantitative variables, according to the distribution. The qualitative variables were described as absolute and relative frequencies.

c. For inferential statistics, the groups were compared (left vs. right radial route)

d. with Student's T or Mann-Whitney U for quantitative variables (according to distribution);

e. Chi square was used for qualitative variables.

f. A value of $\mathrm{p}<0.05$ was considered statistically significant.

\section{Ethical aspects}

i. This protocol establishes a minimum risk investigation according to the Regulations of the Law of General Health in Matters of Health Research, since questionnaires of written way.

ii. The investigation procedures were within the framework of the Regulations of the General Health Law on Health Research and the Declaration of Helsinki.

iii. This study has an informed consent form, which was applied after the personalized explanation of the protocol's objective and prior to carrying out questionnaires.

iv. The contributions of this study to participants and society lie in the progress knowledge of comfort in performing these procedures, both for the patient as for the operator.

v. The information obtained as part of this study is strictly confidential.

\section{Results}

From January 2019 to September 2019, 64 participants were enrolled, of which 9 were excluded ( 3 patients with chronic kidney disease, 6 who refused to participate), leaving 55 patients who were randomized (age $62 \pm 12$ years, men 78\%), 27 were randomized to the coronary angiography by left radial access and 28 by right radial access coronary angiography. 23 of the left approaches were made at the snuffbox $(83 \%, \mathrm{p}=0.001)$ and 4 at the wrist level; on the other hand 11 of the right radial approaches were performed at the distal level and 17 at the wrist level. The indications to carry out coronary angiography were: ischemic heart disease (the most frequent) $(60 \%$ left, vs $43 \%$ right), acute coronary syndromes without ST-segment elevation were the second more frequent indication (22\% left radial vs. $32 \%$ right radial), followed by acute coronaries with ST segment elevation (7\% left radial vs. $11 \%$ right radial) and in fourth place was coronary angiography prior to cardiac surgery (11\% left vs $7 \%$ right), without statistically significant difference (Table 1). In relation to the number of conventional projections for left and right coronary system there was no difference (left system $8 \pm 1.9$ vs $8.5 \pm 1.7$ right system $\mathrm{p}=0.140$ ) (Table 2). Regarding the primary outcome comfort before the procedure, during the procedure and after most of the patients stated to agree (4=Agree), without existing Statistical difference in this regard (Table 3 ). In the bleeding analysis there was no difference, only 4 patients presented bleeding BARC 1 (left $7 \%$ vs right $7 \%$, $\mathrm{p}=0.99)$, the spasm presentation was $11 \%$ and $26 \%$ respectively $(\mathrm{p}=0.177)$. Artery spasm $26 \%$ for left ( 4 snuff box, 3 wrist) and $11 \%$ for right (2 snuff box, 1 wrist).

Table I Baseline characteristics

\begin{tabular}{llll}
\hline & Right approach & Left approach & P value \\
\hline Age & $60 \pm 11$ & $65 \pm 13$ & 0.18 \\
Male & $82 \%$ & $74 \%$ & 0.469 \\
Snuffbox & $39 \%$ & $85 \%$ & 0.001 \\
Indication & $18 \%$ & & \\
STEMI & $32 \%$ & $7 \%$ & \\
$\begin{array}{l}\text { NSTEMI } \\
\begin{array}{l}\text { Stable coronary } \\
\text { disease }\end{array}\end{array}$ & $43 \%$ & $22 \%$ & \\
$\begin{array}{l}\text { Before cardiac } \\
\text { surgery }\end{array}$ & $7 \%$ & $60 \%$ & 0.455 \\
\hline
\end{tabular}

Table 2 Procedure characteristics

\begin{tabular}{llll}
\hline & Right approach & Left approach & P value \\
\hline $\begin{array}{l}\text { Left coronary } \\
\text { system }\end{array}$ & & & \\
LAO & $61 \%$ & $85 \%$ & 0.068 \\
RAO & $57 \%$ & $44 \%$ & 0.346 \\
RAO caudal & $100 \%$ & $96 \%$ & 0.491 \\
RAO cranial & $71 \%$ & $67 \%$ & 0.775 \\
Left PA & $100 \%$ & $85 \%$ & 0.051 \\
PA caudal & $75 \%$ & $70 \%$ & 0.700 \\
LAO craneal & $93 \%$ & $85 \%$ & 0.669 \\
LAO caudal & $100 \%$ & $88 \%$ & 0.105 \\
Lateral & $4 \%$ & $7 \%$ & 0.611 \\
Right coronary & & & \\
LAO & $100 \%$ & $93 \%$ & 0.236 \\
RAO & $36 \%$ & $30 \%$ & 0.631 \\
PA craneal & $78 \%$ & $58 \%$ & 0.117 \\
General & & & 0.140 \\
Number coronary & $8.7 \pm 1.5$ & & \\
Projections & & & \\
\hline & & & \\
\hline
\end{tabular}


Table 3 Results

\begin{tabular}{llll}
\hline & Right approach & Left approach & P value \\
\hline Pre procedure comfort & $4.0 \pm 0.67$ & $4.1 \pm 0.75$ & 0.569 \\
Procedure comfort & $3.93 \pm 0.77$ & $4.2 \pm 0.68$ & 0.196 \\
Post procedure comfort & $4.18 \pm 0.62$ & $4.29 \pm 0.82$ & 0.549 \\
Contrast $(\mathrm{ml})$ & $88 \pm 55$ & $103 \pm 85$ & 0.436 \\
Distance $(\mathrm{cms})$ & $47.6 \pm 4.6$ & $47.7 \pm 4.8$ & 0.941 \\
Number of catheters & $1.4 \pm 0.7$ & $1.5 \pm 0.6$ & 0.348 \\
Fluoroscopy time (min) & $12.3 \pm 6.5$ & $14.9 \pm 15.0$ & 0.411 \\
Procedure time & $32.3 \pm 19.8$ & $31.3 \pm 20.7$ & 0.862 \\
Change in approach & $4 \%$ & $22 \%$ & 0.051 \\
Secondary outcomes & & & \\
Bleeding & $7 \%$ & $7 \%$ & $>0.99$ \\
Artery spasm & $11 \%$ & $26 \%$ & 0.177 \\
\hline
\end{tabular}

The amount of contrast used for diagnostic studies was $88+55 \mathrm{ml}$ for the right radial and $103+55 \mathrm{ml}$ for the left radial $(\mathrm{p}=0.436)$ (Figure 8). The distance between intensifier-researcher was $47.6 \mathrm{~cm}+4.6$ in coronary angiography performed at the right side and $47.7 \mathrm{cms}+4.8$ for those made on the left side ( $\mathrm{p}=0.941$ ) (Figure 9). The number of catheters was $1.4+0.77$ in procedures on the right side and $1.5+0.6$ for left $(\mathrm{p}=0.348)$. Fluoroscopy times expressed in minutes were $12.3 \mathrm{~min}$ +6.5 for right procedures and $14.9 \mathrm{~min}+15$ for left procedures $(0.411)$, the time total of the diagnostic procedure was $32.3 \mathrm{~min}+19.8$ in right angiography and $31.3 \mathrm{~min}+20.7$ for left angiograms (0.862). Finally, an access change was made in $1(4 \%)$ right radial procedure, which was performed in the anatomic snuffbox; the change was due to an abnormal origin of the right coronary artery and opted to change to the femoral route. On the other hand, in $4(22 \%)$ of the procedures performed by the left side, access site was changed: it was found that in 1 patient it was not possible perform distal puncture and the operator proceeded to perform it at the wrist; in another patient the operator could not obtain distal access either and right radial access was performed with a conventional technique; and in the last 2, a femoral approach was used after spasm. The $\mathrm{p}$ value for the difference of change of right vs. left access was $=0.051$.

\section{Discussion}

This is the first study to randomly assess left radial approach versus right radial approach, in patients who undergo coronary angiography introducing a novel comparison of patient comfort, an aspect that seems to be of little importance at the time of taking a coronary angiography but that can take significance when procedures become prolonged. In the technical aspect of the procedure, the decision of performing conventional or distal access was not a randomization parameter since not all researchers are trained in both techniques; on both approaches, coronary projections and ventriculography could be performed without finding a significant statistical difference. In the left technique catheters as Judkins and TIG were used despite the fact that one of the advantages of performing a left-side approach is the use of Judkins catheters to take advantage of support mechanics on the aortic wall. The cases in which angioplasty was performed were not studied in the present investigation, since the procedure time of the fluoroscopy varies depending on the scenario and we tried to compare both techniques in a simple way during the diagnostic moment of coronary procedures.

The comfort aspect is a very subjective parameter for which there is no specific scale of measurement. A Likert scale was used to rate the parameter from 1 to 5 , from being strongly disagree (1), disagree (2), be indifferent (3), agree (4) and (5) strongly agree, during the study the majority of patients rated agreeing that the two types of access are comfortable before, during and after the procedure without finding a statistically significant difference which contradicts our hypothesis that assumed the left access as more comfortable. For the purposes of this study, the perception of comfort between wrist levels or in the anatomic snuffbox was not investigated, and it could be reason for future research. Both access routes were safe, only 4 patients presented small bruises at the puncture site that did not warrant medical attention; we had more cases with artery spasm in the left access group compared to the right, this is probably related to the learning curve for the different techniques, despite this there was no difference that had statistical significance. Within the rest of the objectives there was a strong tendency to change the access site in left procedures almost reaching statistical significance, in two cases after attempting distal puncture and not being able to obtain the access conventional puncture was done, there were also two cases were spasm was presented and operators changed to the femoral route. In the right access only one change to the femoral route was made because an anomalous origin of right coronary artery emerging from the left valsalva sinus made difficult to cannulate by radial approach, this phenomenon possibly is also related to the learning curve of the left techniques, also the researchers.

Fluoroscopy times were slightly higher but without significant difference for the left accesses. It was striking that there was no great difference in the distances between the patient and the operator.

\section{Limitations}

The present study was carried out in 2 institutions with different types of population and in 2 coronary Cath labs that have different human and material resources, for this reason patient recruitment from the Instituto Nacional Salvador Zubirán was limited. The technique left radial puncture was not standardized and the position at the time 
of puncture was differently, one investigator performed the puncture positioned on the patient's right side and accommodating the left arm on the abdomen thus performing the puncture, while the rest of the investigators chose to puncture the patient's left side once access was accommodated the patient with the arm over the abdomen and started the procedure on the right side.

Initially, the acquisition of a radiation dosimeter was considered, however, when having patients of different centers could not homogenize the radiation measurements since each center has assigned different dates to obtain said reading, it was decided to have a simple and indirect method to assess the exposure so the distance between the intensifier and the operator left shoulder was the defined method for assessing radiation, however our measurement is not a parameter reliable nor is it known what the specific correlation is with the amount of radiation.

\section{Conclusions}

The present study shows that the left radial access made distally or in the carpal region is safe in terms of bleeding or spasm when compared to right access approach. Judkins diagnostic catheters and TIG catheters can be used despite differences in support mechanics and cannulation maneuvers, and ventriculography is feasible. In the other hand is not an access that has been shown to be superior in comfort despite that in right technique the arm is slightly abducted at the time of the procedure. Left approach can be used in different clinical settings for either elective or emergency procedures, and is a valuable alternative either to be used as a first choice or as an option secondary when no good pulses are found on the right side or when you want to protect the integrity of the right radial artery to be used as a vascular graft in surgery of revascularization.

\section{Acknowledgments}

None.

\section{Conflicts of interest}

Author declares that there are no conflicts of interest.

\section{Funding}

None.

\section{References}

1. Forssmann W. Die Sondierung des Rechten Herzens. Klin Wochenschr. 1929;8:2085-2087.
2. Regato JA. In Memoriam. Pedro L. Farinas, edirors. Radiology. 1951;57(1):110-111.

3. Seldinger SI. Catheter replacement of the needle in percutaneous arteriography; a new technique. Radiol Act. 1953;39(5):368-376.

4. Fogarty TJ, Daily PO, Shumway NE, et al. Experience with balloon catheter technique for arterial embolectomy. Am J Surg. 1971:122(2):231237.

5. Dotter CT, Judkins MP. Transluminal treatment of arteriosclerotic obstruction. Description of a new technique and a preliminary report of its application. Circulation.1964;30:654-670.

6. Grüentzig AR, Senning Å, Siegenthaler WE. Nonoperative dilatation of coronary-artery stenosis: percutaneous transluminal coronary angioplasty. $N$ Engl J Med. 1979;301(2):61-68.

7. Grier D, Hartnell G. Percutaneous femoral artery puncture: practice and anatomy. Br J Radiol. 1990;63(752):602-604.

8. Kiemeneij F, Laarman GJ, Odekerken D, et al. A randomized comparison of percutaneous transluminal coronary angioplasty by the radial, brachial and femoral approaches: the access study. J Am Coll Cardiol. 1997;29(6):1269-1275.

9. Campeau L. Percutaneous radial artery approach for coronary angiography. Cathet Cardiovasc Diagn. 1989;16(1):3-7.

10. Kiemeneij F, Laarman GJ, de Melker E. Transradial artery coronary angioplasty. Am Heart J. 1995;129(1):1-7.

11. Kiemeneij F. Left distal transradial access in the anatomical snuffbox for coronary angiography (ldTRA) and interventions (ldTRI). EuroIntervention. 2017;13(7):851-857.

12. Hadjivassiliou A, Kiemeneij F, Nathan S, et al. Ultrasound-guided access of the distal radial artery at the anatomical snuffbox for catheterbased vascular interventions: A technical guide. EuroIntervention. 2019; EIJ-D-19-00555.

13. Kim Y, Ahn Y, Kim I, et al. Feasibility of coronary angiography and percutaneous coronary intervention via left snuffbox approach. Korean Circ J. 2018;48(12):1120-1130.

14. Mizuguchi Y, Izumikawa T, Takahashi A, et al. TCT-846 Multicenter Study of the Efficacy and Safety of Distal Radial Approach in Coronary Angiography and Intervention. Journal of the American College of Cardiology. 2018;72(13).

15. Koutouzis M, Kontopodis E, Tassopoulos A, et al. Hand Hematoma After Cardiac Catheterization Via Distal Radial Artery. J Invasive Cardiol. 2018;30(11):428-428. 